\title{
Blunted Cardiac AMPK Response is Associated with Susceptibility to Ischemia/ Reperfusion in Male Offspring of Gestational Diabetic Rats
}

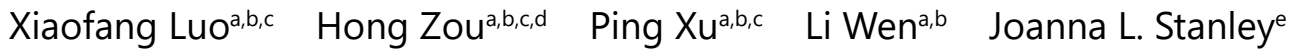 \\ Xiaoxue Jiang ${ }^{f}$ Ting-li Han ${ }^{e}$ David Olsong Chuan Peng ${ }^{b, c} \quad$ Chen Zhang ${ }^{a, b, c}$ \\ Xiaoqiu Xiao $^{b, c}$ Chao Tong ${ }^{a, b, c}$ Hongbo Qja,b,c Philip N. Baker ${ }^{b, h}$
}

aDepartment of Obstetrics, The First Affiliated Hospital of Chongqing Medical University, Chongqing, China, 'International Collaborative Joint Laboratory of Reproduction and Development, Ministry of Education, Chongqing Medical University, Chongqing, China, 'State Key Laboratory of Maternal and Fetal Medicine of Chongqing, Chongqing Medical University, Chongqing, China, dDepartment of Obstetrics and Gynecology, University-Town Hospital of Chongqing Medical University, Chongqing, China, eliggins Institute, University of Auckland, Auckland, New Zealand, 'Department of Cardiology, The First Affiliated Hospital of Chongqing Medical University, Chongqing, China, ${ }^{9}$ Departments of Obstetrics and Gynecology, Pediatrics and Physiology, University of Alberta, Edmonton, AB, Canada,

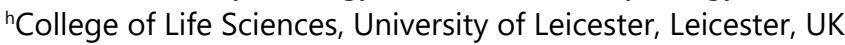

\section{Key Words}

Gestational diabetes mellitus - Fetal programming • AMPK • Ischemia/reperfusion • Cardiac function

\footnotetext{
Abstract

Background/Aims: Gestational diabetes mellitus (GDM) is closely associated with early perinatal complications and long-term health problems, such as cardiovascular disease, in offspring. AMP-activated protein kinase (AMPK) is cardioprotective, particularly in the treatment of ischemia/reperfusion (I/R). However, whether GDM programs offspring susceptibility to cardiac I/R and the involvement of AMPK remain unclear. Methods: Streptozotocin was administered to rats during mid pregnancy; the postpartum maternal metabolome was assessed by chromatography-mass spectrometry (GC-MS). Male offspring were subjected to body composition scanning followed by ex vivo global I/R. Cardiac signaling was determined by Western blotting. Results: The body weights (BWs) of the GDM male offspring were significantly heavier than those of the control group from the age of 8 weeks; the heart weights (HWs) and HW/BW were also increased in the GDM group compared to the control group. The ex vivo post-I/R cardiac contractile function recovery was significantly compromised in

X. Luo, H. Zou and P. Xu contributed equally to this work.

Hongbo Qi, M.D, Ph.D., Department of Obstetrics and Gynecology, The First Affiliated Hospital of Chongqing Medical University Xiaoqiu Xiao Ph.D. $\quad$ No. 1 Youyi Road, Yuzhong District, Chongqing 400016 (China); Tel. +86 23 89011791; +86 23 $89011102 ;$ and Chao Tong Ph.D. + +8623 8901866, E-Mail qihongbo728@163.com; chaotongcqmu@163.com; bshaw2001@163.com
} 


\section{Cellular Physiology Cell Physiol Biochem 2019;52:1103-1116

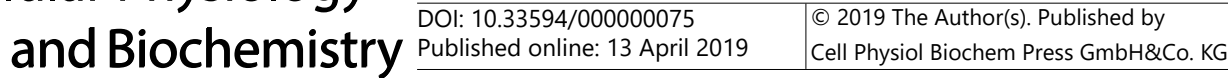 \\ Luo et al.: GDM Impairs Cardiac AMPK in Male Offspring}

the GDM male offspring. The phosphorylation of AMPK and ACC was elevated by ex vivo I/R in both groups, but to a significantly lesser extent in the GDM group. Conclusion: GDM male offspring rats have higher risks of overgrowth and intolerance to cardiac $I / R$, which may be due to a compromised AMPK signaling pathway.

(C) 2019 The Author(s). Published by Cell Physiol Biochem Press GmbH\&Co. KG

\section{Introduction}

Gestational diabetes mellitus (GDM) is defined as glucose intolerance that develops or is first recognized during pregnancy; the condition typically resolves shortly after delivery [1]. The prevalence of GDM is as high as $20 \%$; women of all ethnicities are affected, and the incidence is rapidly increasing worldwide [2], making this condition a major global health concern.

Accumulating evidence indicates that GDM has numerous effects on both the mother and offspring. These effects not only include short-term complications but also consequences for long-term health [3]. Most women diagnosed with GDM have normal glucose concentrations after delivery; however, they remain at a higher risk of developing type 2 diabetes in later life [4]. The development of GDM may have serious consequences for the neonate, including increased risks of developing macrosomia and birth injuries, such as shoulder dystocia, stillbirth, and sudden infant death [5]. Further, studies of individuals born following a pregnancy complicated by GDM have shown that the affected infants are at an increased risk of congenital malformations, hypoglycemia, respiratory distress syndrome, polycythemia and hypertrophic cardiomyopathy in the immediate postnatal period [6-9]. Evidence from long-term studies suggests that offspring are also at increased risks of developing obesity, diabetes, and cardiovascular disease in adulthood [10-12].

Adenosine monophosphate-activated protein kinase (AMPK), a serine/threonine kinase, plays an important role in cellular energy metabolism homoeostasis and is expressed in many tissues and cell types, including cardiomyocytes $[13,14]$. AMPK attenuates high glucoseinduced oxidative stress in heart tissue and consequent cardiomyocyte apoptosis [15]; thus, it may be a potential cross-species target of cardiac function in diabetes [16]. However, the interplay of fetal cardiac AMPK and intrauterine hyperglycemia is poorly understood, with limited information available regarding the role of AMPK in the programming of offspring cardiac function. Given its ability to act in a protective role in cardiomyocytes in a hyperglycemic environment, the aim of this study was to investigate the involvement of AMPK in abnormal fetal cardiac development in a hyperglycemic intrauterine environment.

\section{Materials and Methods}

\section{Establishment of a rat GDM model}

Sixteen female Sprague-Dawley (SD) rats, which weighed 220 250 g at the age of 8 weeks, were mated with 10-week-old male SD rats (250 300 g). Animals were obtained from the Experimental Animal Center of Chongqing Medical University. The animal experiments were approved by the Medical Ethics Committee of Chongqing Medical University. After overnight mating, a sperm-positive vaginal smear the next morning indicated pregnancy and was noted as gestational day (G) 0.5. Pregnant rats were randomly assigned to the GDM or control groups (8 rats per group). GDM was induced by STZ (35 mg/kg body weight, Sigma, St. Louis, MO, USA), administered via intraperitoneal (i.p.) injection on G8.5. The rats in the control group received an injection of citrate buffer under the same conditions. The blood glucose concentration was measured in both groups on G8.5, followed by every three days; the GDM rat model was deemed successfully established, as the average causal blood glucose level was $\geq 16.7 \mathrm{mmol} / \mathrm{L}$. A small drop of blood was obtained from the tail vein, and the blood glucose concentration was measured using a glucometer (ACCU-CHEK Integra, Roche, Germany). The rats were then allowed to deliver spontaneously. All rats were maintained in controlled temperature conditions $\left(22-25^{\circ} \mathrm{C}\right)$, relative humidity (60\%), and a $12 \mathrm{~h}$ light-dark cycle (lights on at 08:00). All male offspring rats were provided with free access to a standard diet and tap water after weaning. 


\section{Cellular Physiology Cell Physiol Biochem 2019;52:1103-1116

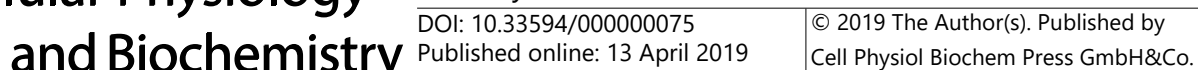 \\ Luo et al.: GDM Impairs Cardiac AMPK in Male Offspring}

Glucose tolerance test (GTT) and insulin tolerance test (ITT)

GTT and ITT assays were performed as previously established in our laboratory [17]. Briefly, male offspring were fasted overnight with free access to tap water before the GTT test. The blood collected from tail vein in conscious was used to determine the blood glucose concentration using a glucometer (ACCUCHEK Integra, Roche, Germany). This was deemed the 0 min sample. To perform a GTT, the rats then received a glucose bolus ( $2 \mathrm{~g} / \mathrm{kg}$ body weight, i.p.), and the blood glucose concentration was measured at 15, 30, 60, and 120 mins.

The rats were fasted for 4 hours with free access to tap water before the ITT test. The blood glucose concentration was measured, as previously described, before the rats received an insulin bolus (1 IU/ kg body weight, i.p.). The blood glucose concentrations were then measured at 30, 60, 90 and 120 mins postinjection.

\section{ELISA}

The serum levels of insulin (INS) in the rats were detected by commercial ELISA kits purchased from Shanghai Hu Shang Biological Technology Co., Ltd. (Shanghai, China) in accordance with the manufacturer's instructions. In brief, the microplates were coated with INS antibodies at $4{ }^{\circ} \mathrm{C}$ overnight, and the standard and serum were subsequently combined with HRP-labeled INS antibodies at $37^{\circ} \mathrm{C}$ for 1 hour; after washing completely, tetramethylbenzidine (TMB) substrate was added for colorimetric measurement of INS. The absorbance (OD) at $450 \mathrm{~nm}$ was detected by a microplate reader; the concentrations of INS were calculated using standard curves (logistic).

Metabolomic analyses by Chromatography-Mass Spectrometer (GC-MS)

Metabolomic analyses of maternal serum were performed as previously reported [18]. Methyl chloroformate (MCF) derivatives were detected in an Agilent Technology GC system (7890B, Agilent Technology, California, USA) coupled to an MSD mass selective detector (EI) (5977A, Agilent Technology, California, USA) set at $70 \mathrm{eV}$. ZB-1701 GC capillary columns $(0.15 \mu \mathrm{m}$ x $250 \mu \mathrm{m}$ id $\times 30$ min with $5 \mathrm{~m}$ guard column, Phenomenex) were used for all tests. All samples were injected with the injector temperature at $290^{\circ} \mathrm{C}$ under pulsed splitless mode. A constant flow of $1 \mathrm{~mL} / \mathrm{min}$ of helium gas flow was set through the GCcolumn. The GC-oven temperature was originally held at $45^{\circ} \mathrm{C}$ for $2 \mathrm{~min}$, followed by a gradient ascent of 9 ${ }^{\circ} \mathrm{C} / \mathrm{min}$ to $180^{\circ} \mathrm{C}$; after $5 \mathrm{~min}$, the temperature raked at $40^{\circ} \mathrm{C} / \mathrm{min}$ to $220^{\circ} \mathrm{C}$. After an additional $5 \mathrm{~min}$, the temperature was ascent with a gradient of $40^{\circ} \mathrm{C} / \mathrm{min}$ to $240^{\circ} \mathrm{C}$ for $11.5 \mathrm{~min}$; ultimately, the temperature was sloped up $40^{\circ} \mathrm{C} / \mathrm{min}$ until it reached $280^{\circ} \mathrm{C}$ and remained at $280^{\circ} \mathrm{C}$ for $7 \mathrm{~min}$. The ion source temperature was set to $230^{\circ} \mathrm{C}$, the quadrupole temperature was set at $150^{\circ} \mathrm{C}$ and the interface temperature was $250^{\circ} \mathrm{C}$. The mass spectrometry was conducted in scan mode and began after $5.5 \mathrm{~min}$ with scan rate of $0.1 \mathrm{sec}$ and a mass range between 38 and $550 \mathrm{amu}$.

\section{Dual energy X-ray absorptiometry (DXA)}

The DXA scans were performed as previously described with modification [19]. In brief, the animals were anesthetized using chloral hydrate $(10 \%, 3 \mathrm{ml} / \mathrm{kg}$ body weight, i.p.). DXA scans were performed with a fan beam QDR 4500 A densitometer (Hologic, Inc., Bedford, MA, USA) calibrated daily in accordance with the manufacturer's recommendations. The animals were positioned centrally at the bottom of a square. All DXA measurements and analyses were performed by the same investigator, and the data were analyzed using the small-animal mode of Hologic Discovery Software (Hologic, Inc., Bedford, USA).

\section{Measurement of blood pressure (BP) and heart rate (HR)}

BP and HR were assessed by tail-cuff plethysmography (BP-2000 Blood pressure Analysis system, Visitech System, NC, USA) in conscious rats. The rats were trained to be adaptive to the apparatus for 7 consecutive days, with measurements carried out on the following 7 days. The rats were maintained in the restrainers for 5 to 10 preliminary measurements before the actual measurements were initiated, with 10 to 20 actual measurements obtained in each session. 


\section{Cellular Physiology Cell Physiol Biochem 2019;52:1103-1116 \\ \begin{tabular}{ll|l} 
and Biochemistry $10.33594 / 000000075$ & $\begin{array}{l}\text { Published online: } 13 \text { April } 2019 \\
\text { Cell Physiol Biochem Press GmbH\&Co. KG }\end{array}$ \\
\hline
\end{tabular} \\ Luo et al.: GDM Impairs Cardiac AMPK in Male Offspring}

\section{Ex vivo cardiac I/R model}

An ex vivo cardiac I/R model was established as previously described [20]. Briefly, 24-week-old male offspring were anesthetized using chloral hydrate $(10 \%, 3 \mathrm{ml} / \mathrm{kg}$ body weight, i.p.), 10 minutes after a heparin injection $\left(1000 \mathrm{U} / \mathrm{kg}\right.$, i.p.). The hearts were rapidly excised and immersed in prechilled $\left(4^{\circ} \mathrm{C}\right) \mathrm{Krebs}-$ Henseleit bicarbonate buffer (KHB; pH 7.4, which contained in mmol/L $118 \mathrm{NaCl}, 1.2 \mathrm{KH}_{2} \mathrm{PO}_{4}, 4.7 \mathrm{KCl}, 1.4$ $\mathrm{CaCl}_{2}, 1.2 \mathrm{MgSO}_{4}, 0.4$ sodium oleate, 11 glucose, $10 \mathrm{uU} / \mathrm{ml}$ insulin and $1 \% \mathrm{BSA}$ ). Isolated hearts were then subjected to ex vivo I/R via the use of the Langendorff Heart Perfusion System (Adnoti Glass Technology, Inc. Monrovia, CA, USA) and perfused by $37^{\circ} \mathrm{C}$ KHB that was continuously infused with oxygen (95\%) and carbon dioxide (5\%). The perfusion pressure was maintained at $70 \mathrm{mmHg}$. The contractile function was measured and recorded by a Physiological counter (Biopac system, Goleta, CA, USA), and the data were analyzed by Powerlab software (ADInstrument, CA, USA).

\section{Immunoblotting}

Immunoblotting was performed as previously reported [20]. Briefly, the left ventricular tissue or liver tissue was rapidly removed and homogenized with ice-cold RIPA lysis buffer (Thermo Pierce Biotechnology, Rockford, IL, US). The protein concentration was determined using a Bradford protein assay kit (Takara, Shiga, Japan). Twenty micrograms of total protein in each sample were resolved on polyacrylamide gel, transferred onto a polyvinylidene fluoride (PVDF) membrane (Millipore, Billerica, MA, USA) and blocked with $5 \%$ nonfat dried milk powder (Bio-Rad, CA, USA). The membranes were then incubated overnight at $4{ }^{\circ} \mathrm{C}$ with the primary antibodies of anti-ERK, anti-p-ERK, anti-Akt, anti-p-Akt, anti-AMPK, anti-p-AMPK, anti-ACC, anti-p-ACC, anti-JNK, anti-mTOR (Cell Signaling Technology, Danvers, MA, USA), or anti-PPAR $\gamma$, anti-p-PPAR $\gamma$, anti-p-JNK (Santa Cruz Biotechnology, Santa Cruz, CA, USA), or anti-p-mTOR (Abcam, Cambridge, MA, USA). The membranes were subsequently washed and incubated with secondary antibody linked to horseradish peroxidase (Santa Cruz, CA, USA). Images were acquired using ChemiDoc (Bio-Rad, CA, USA). The reagents for Western blot detection by enhanced chemiluminescence (ECL) were purchased from Millipore (Billerica, MA, USA), and the density of the bands was measured with Quantity One System (Bio-Rad, Richmond, CA, USA).

\section{Statistical analysis}

Data are expressed as the mean \pm SEM, and significance was tested by Student's unpaired two-tail $t$ tests, with $\mathrm{p}<0.05$ considered significant. Repeated measures ANOVA with Mauchly's test for sphericity and a multivariate test were used to determine the differences in the maternal blood glucose, maternal body weight, male offspring body weight, male offspring blood glucose, GTT, and ITT results; a value of $\mathrm{p}<0.05$ was considered statistically significant. Statistical analysis was performed using GraphPad Prism 5.0 software (GraphPad Software, La Jolla, CA, USA) and SPSS 17.0 statistical software (SPSS Inc., Chicago, IL, USA).

\section{Results}

STZ induced hyperglycemia and metabolic disturbance in pregnant rats

The blood glucose of the control group ranged from $4.83 \pm 0.24 \mathrm{mmol} / \mathrm{L}$ to $5.87 \pm 0.16$ $\mathrm{mmol} / \mathrm{L}$; however, it was significantly higher in the GDM group starting from G11.5 (3 days after STZ treatment) and reached a peak of $25.90 \pm 1.40 \mathrm{mmol} / \mathrm{L}$ at G14.5 (p<0.05; Fig. 1A). The blood glucose level in the GDM group remained significantly higher for the remainder of gestation, whereas it decreased to $12.88 \pm 3.91 \mathrm{mmol} / \mathrm{L}$ on the $21 \mathrm{st}$ day postpartum. Consistently, the plasma insulin level was significantly lower in the STZ group (Fig. 1B). In addition, the body weight of the STZ-treated dams was significantly lower in late pregnancy $(\mathrm{p}<0.05$; Fig. 1C).

The impact of STZ on maternal metabolism, which might potentially influence offspring, was investigated. Metabolic profiling of perinatal blood samples was performed by GCMS. The results indicated over 177 chromatographic peaks (potential metabolites) that were relatively different between the GDM and controls (Fig. 1D). Among these peaks, 22 metabolites, including tricarboxylic acid cycle (TCA) intermediates, vitamins, amino acids, saturated fatty acids and unsaturated fatty acids, were identified using an in-house mass 


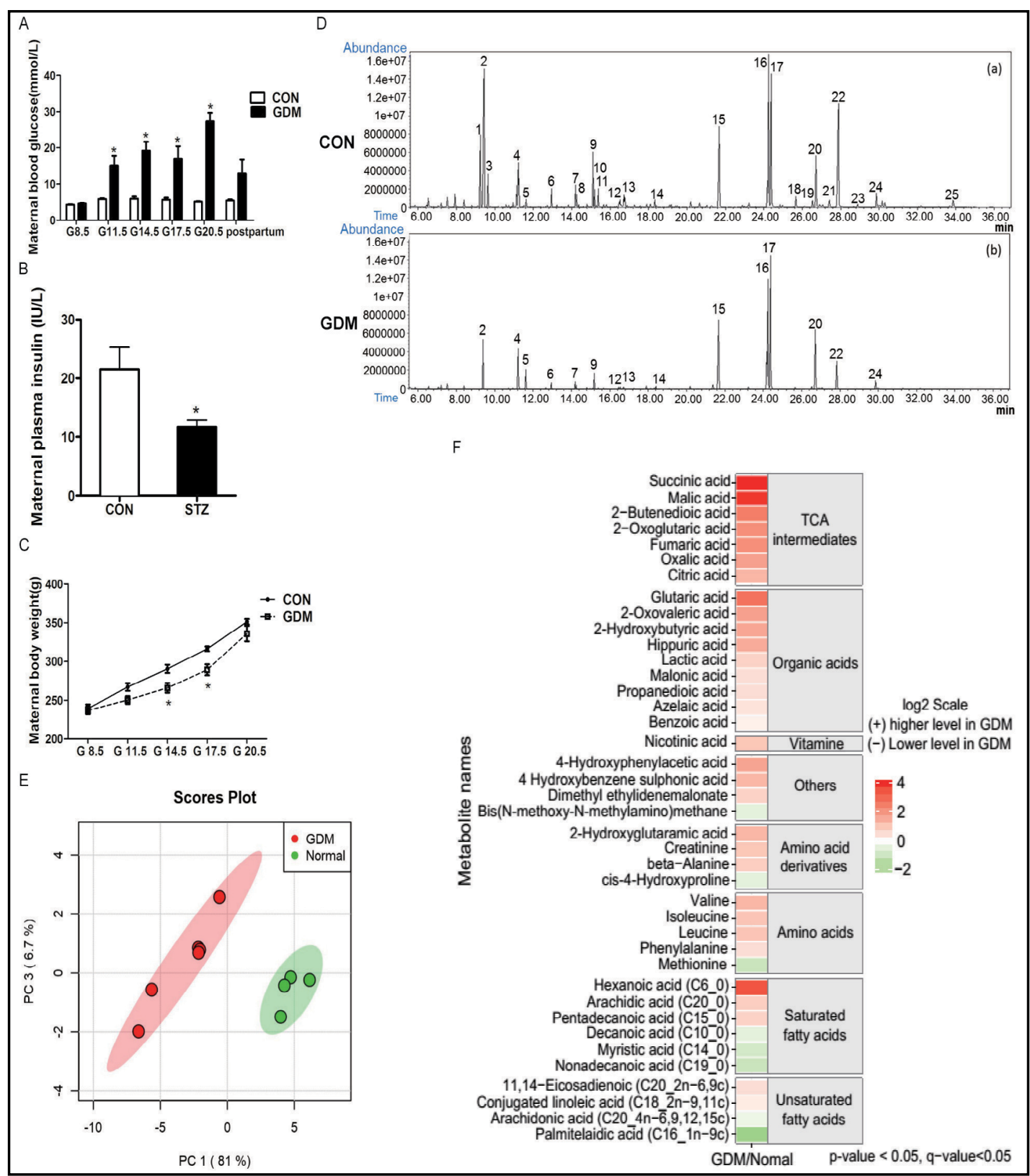

Fig. 1. STZ induced hyperglycemia and metabolic disturbance in pregnant rats. (A) Maternal blood glucose levels, (B) maternal plasma insulin on G17.5, (C) maternal body weight after STZ treatment, (D) representative GC-MS chromatogram of plasma metabolite profile of mice with (a) GDM and (b) normal pregnancy. Succinic acid (Peak 1), lactic acid (Peak 2), Caprylic acid (Peak 3), D4-alanine (Peak 4- internal standard), glycine (Peak 5), valine (Peak 6), leucine (Peak 7), isoleucine (Peak 8), silane (Peak 9), proline (Peak 10), pentanedioic acid (Peak 11), citric acid (Peak 12), asparagine (Peak 13), glutamate (Peak 14), 10,13-dimethyltetradecanoic acid (Peak 15), conjugated linoleic acid (Peak 16), stearic acid (Peak 17), 2,4,6(1H, 3H, 5H)-pyrimidinetrione (Peak 18), lysine (Peak 19), arachidonic acid (Peak 20), histidine (Peak 21), EDTA (Peak 22), tyrosine (Peak 23), DHA (Peak 24), and ethyl 8-amino-6-methoxycinchoninate (Peak 25), (E) principal component analysis (PCA) of metabolites identified from CON and GDM sera; principal components PC1 and PC3 explicate $81 \%$ and $6.7 \%$ of the variance, respectively, (F) differences in maternal plasma metabolome. The results are the mean \pm SEM, $n=4$ in CON and $n=6$ in GDM, ${ }^{*} p<0.05$. 


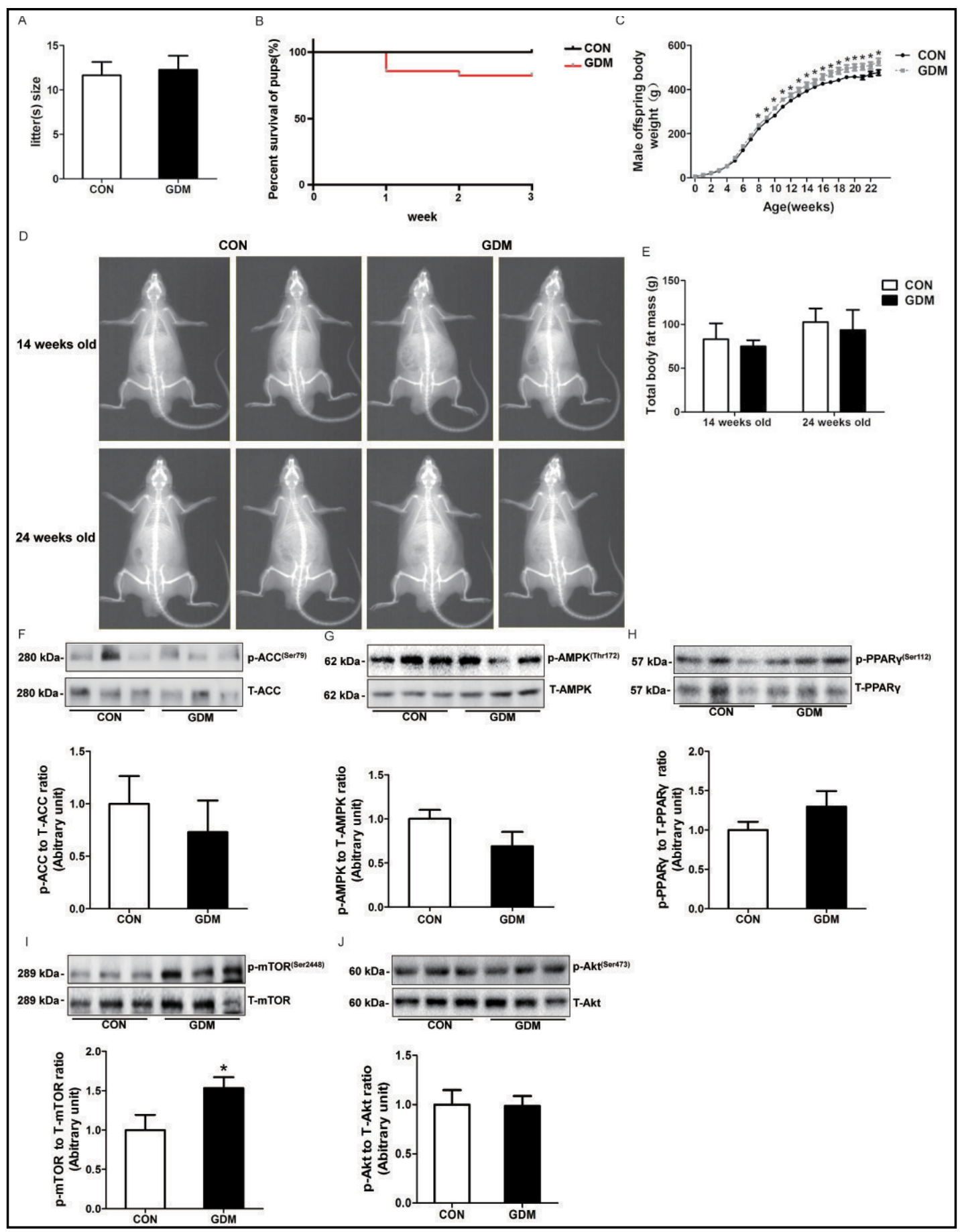

Fig. 2. GDM male offspring rats are associated with accelerated growth after birth. (A) Litter size, (B) survival rate of all pups, (C) body weight of male offspring rats, (D) representative DXA scans of male offspring rats, (E) estimated total fat mass of male offspring rats. Results represent the mean \pm SEM; $n=6$ per group. * p<0.05 vs. CON. Male offspring liver (F) p-ACC, ACC; (G) p-AMPK, AMPK; (H) p-PPAR $\gamma$, PPAR $\gamma$; (I) p-mTOR, mTOR; (J) p-Akt and Akt were determined by Western blotting. The results are the mean \pm SEM; $n \geq 3$ per group. ${ }^{*} \mathrm{p}<0.05$ vs. CON. 
spectrometry library and demonstrated significant changes in the GDM compared to the controls (Fig. 1E). These findings indicate that maternal energy metabolism, amino acid metabolism, lipid metabolism and carbohydrate metabolism were significantly modified by GDM. Most importantly, we found that methionine, an essential amino acid, was significantly reduced in the GDM dams $(n=6, p<0.05)$, which suggests that offspring fostered by a GDM mother may undergo malnutrition.

\section{GDM male offspring rats are associated with accelerated growth after birth}

The average litter size was $11.5 \pm 0.50$ in the control group and $12.5 \pm 0.75$ in the GDM group (Fig. 2A, n=8, p=0.38). Moreover, GDM resulted in neonatal death within 3 weeks after birth (Fig. 2B). Although no difference in birth weight (BW) was identified in the male offspring between the control and GDM groups $(6.62 \pm 0.09 \mathrm{~g}$ vs. $6.47 \pm 0.06 \mathrm{~g}$, respectively, $n=42$ from 8 dams per group, $p=0.14$ ), the offspring born to mothers with GDM were significantly heavier at 8 weeks of age $(\mathrm{p}<0.05)$ (Fig. 2C). Moreover, they remained significantly heavier throughout the remainder of the study. To investigate whether the extra weight gain of the GDM male offspring is derived from an upregulation of adiposity, the body composition was determined by DXA scanning (Fig. 2D). The data indicated that the estimated total fat weight was $93.55 \pm 22.95 \mathrm{~g}$ vs. $74.90 \pm 7.00 \mathrm{~g}(\mathrm{n}=4, \mathrm{p}=0.29)$ in the control and GDM male offspring, respectively, at 14 weeks of age and increased to $102.7 \pm 15.50 \mathrm{~g}$ and $83.10 \pm 18.10 \mathrm{~g}$, respectively, $(\mathrm{n}=4, \mathrm{p}=0.43)$ at 24 weeks of age (Fig. 2E), which indicates that GDM does not lead to abnormal adiposity in male offspring rats. To further confirm this finding, liver tissue from male offspring was subjected to assessment of metabolic signaling pathways. The results demonstrated that, GDM didn't show any effects on neither AMPK-ACC pathway nor the lipogenic signal PPAR $\gamma$, but significantly enhanced m-TOR activation (Fig. $2 \mathrm{~F}-\mathrm{J})$. Taken together, these data strongly suggest that GDM male offspring is associated with overgrowth rather than obesity after birth.

\section{GDM did not influence insulin sensitivity in male offspring rats}

The circulating insulin levels in the male offspring rats were $27.23 \pm 1.21 \mathrm{mU} / \mathrm{L}$ and $29.01 \pm 1.36 \mathrm{mU} / \mathrm{L}$ in the control and GDM groups, respectively $(\mathrm{n}=8, \mathrm{p}=0.35$, Fig. 3A). Consistently, there were no significant differences in the GTT (AUC 1648 \pm 73.09 vs. $1510 \pm 158.7$, respectively, $\mathrm{n}=6, \mathrm{p}=0.45$ ) or ITT (AUC $441.8 \pm 35.91$ vs. 486.8 \pm 43.98 , respectively, $n=6, p=0.46$ ) between the male offspring of the two groups at 22 weeks of age (Fig. 3 B, C, D). These data suggested that GDM might not influence insulin sensitivity in male offspring rats.

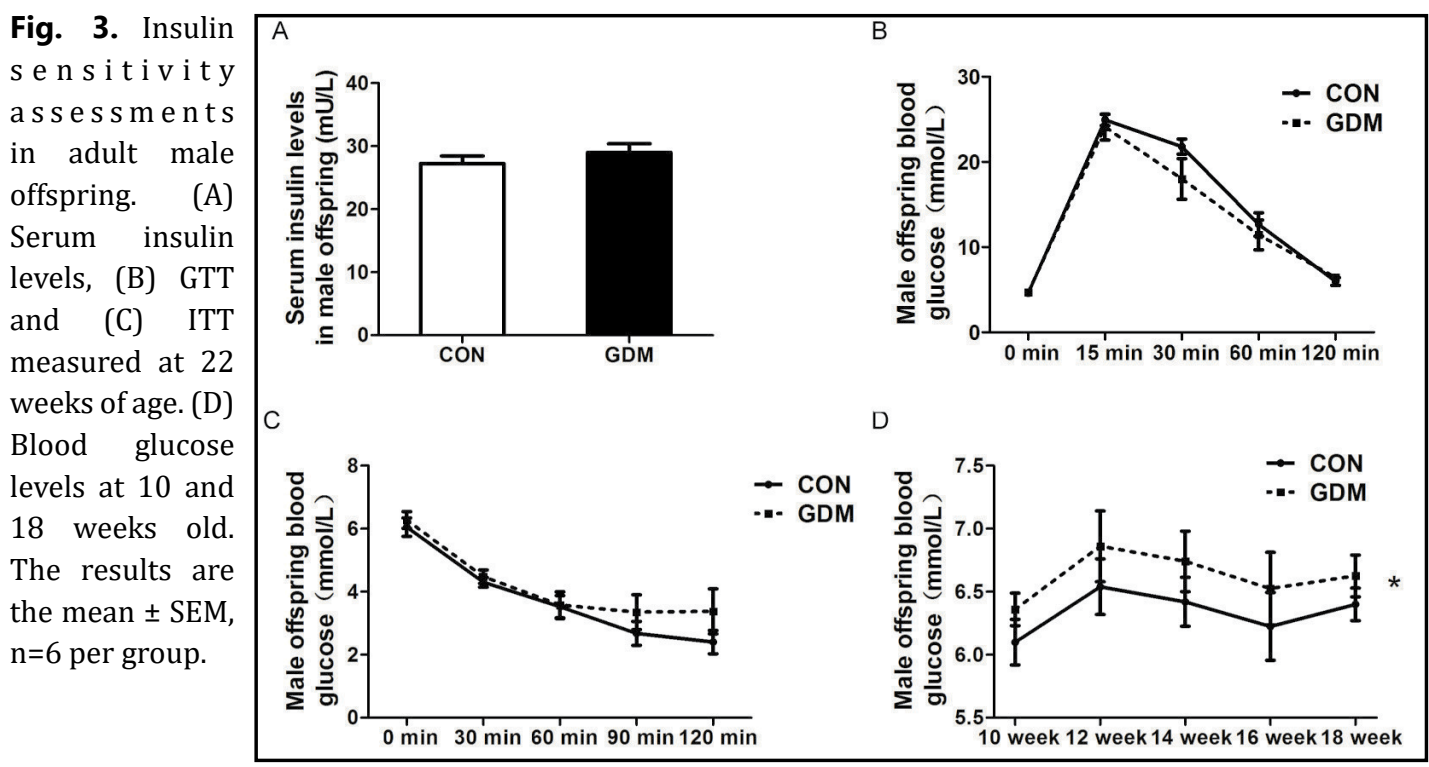




\section{Cellular Physiology Cell Physiol Biochem 2019;52:1103-1116 \begin{tabular}{ll|l} 
and Biochemistry & $\begin{array}{l}\text { DOl: 10.33594/000000075 } \\
\text { Published online: } 13 \text { April 2019 }\end{array}$ & $\begin{array}{l}\text { O 2019 The Author(s). Published by } \\
\text { Cell Physiol Biochem Press GmbH\&Co. KG }\end{array}$ \\
\cline { 2 - 3 }
\end{tabular}

Male offspring rats of GDM pregnancies are associated with cardiac overgrowth

The birth heart weights (HWs) were $0.041 \pm 0.001 \mathrm{~g}$ and $0.038 \pm 0.002 \mathrm{~g}$ in the control and GDM groups, respectively, ( $\mathrm{n}=16$ from 8 dams per group, $\mathrm{p}=0.11$ ), and the heart weight to body weight (HW/BW) ratios at birth were $0.56 \pm 0.01$ and $0.59 \pm 0.03$ in the two groups, respectively ( $n=16$ from 8 dams per group, $p=0.30$ ). However, at 24 weeks of age, the HW of the GDM group was significantly higher than that of the control $(1.48 \pm 0.06 \mathrm{~g}$ vs. $1.26 \pm 0.03 \mathrm{~g}$, respectively, $\mathrm{n}=16$ from 8 dams per group, $\mathrm{p}<0.01$ ). Moreover, the HW/BW was significantly increased in the offspring from the GDM dams at 24 weeks of age $(0.28 \pm 0.01 \mathrm{~g}$ vs. $0.26 \pm 0.003$ $\mathrm{g}$, respectively, $\mathrm{n}=26$ from 8 dams per group, $\mathrm{p}<0.05$ ). Taken together, GDM resulted in cardiac overgrowth in male offspring rats, which may be associated with an altered metabolic signaling pathway and cardiac function.

GDM altered metabolic signaling in hearts of male offspring rats.

Cardiac tissue from male offspring rats was subjected to immunoblotting for AMPK and Akt, both of which are critical metabolic regulators and might be disturbed due to accelerated growth. The data demonstrated that GDM significantly impaired not only Akt but also AMPKACC in the hearts obtained from male offspring (Fig. 4). Moreover, to investigate whether cardiac overgrowth in GDM male offspring is associated with excessive cell division and thus causes duplication induced stress (DIS), mitogen active protein kinases (MAPKase) were determined. The results indicate that GDM compromises cardiac Erk (1/2) phosphorylation in male offspring, whereas it has no influence on JNK. These data suggest that GDM largely influences metabolic signaling in the heart of male offspring.

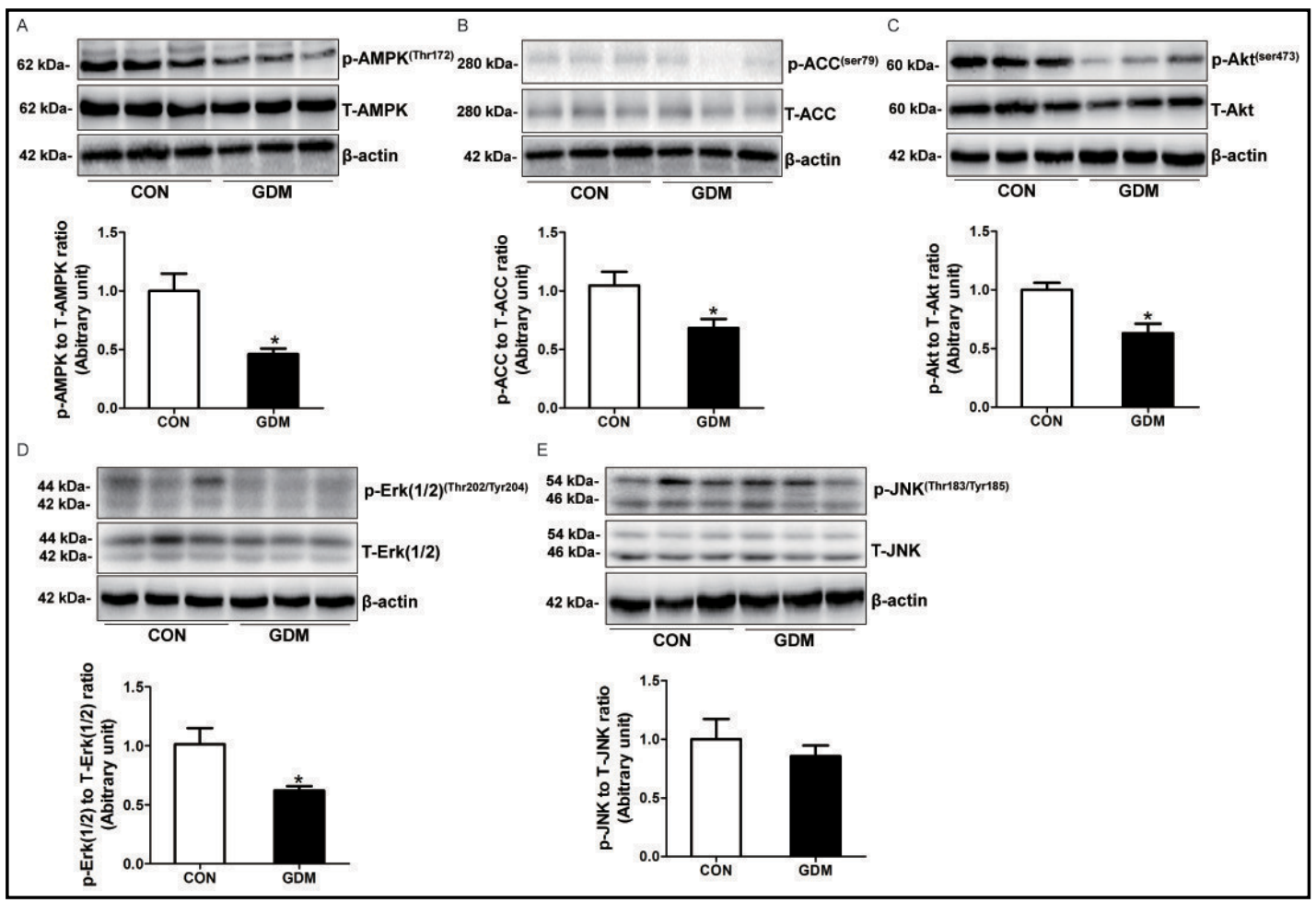

Fig. 4. The effects of GDM on Akt, AMPK-ACC and MAPK in the hearts of offspring. Male offspring cardiac (A) p-Akt, Akt; (B) p-AMPK, AMPK; (C) p-ACC, ACC; (D) p-Erk (1/2), Erk (1/2) and (e) p-JNK, JNK were determined by Western blotting. The results are the mean \pm SEM; $n \geq 4$ per group. ${ }^{*} p<0.05$ vs. CON. 


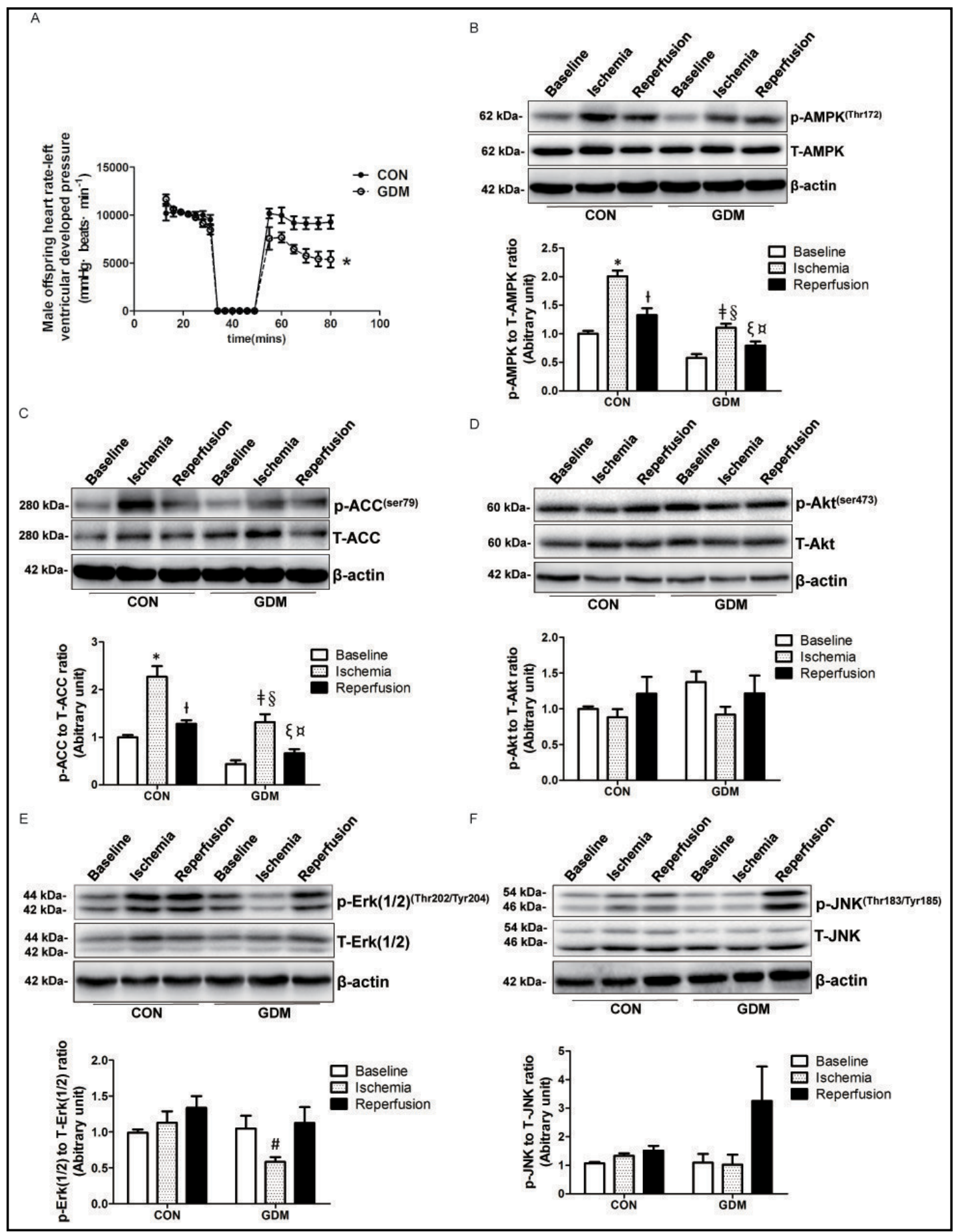

Fig. 5. Altered cardiac signaling pathways in response to I/R in GDM offspring. (A) Hearts were subjected to 20 min of global ischemia followed by 30 min of reperfusion by using the Langendorff perfusion system. Left ventricular contractile function was assessed by HR x LVDP. (B) p-AMPK, AMPK; (C) p-ACC, ACC; (D) p-Akt, Akt; (E) p-Erk (1/2), Erk (1/2) and (F) p-JNK, JNK were determined by Western blotting. Results represent the mean \pm SEM; $n \geq 3$ per group. ${ }^{*} \mathrm{p}<0.05 \mathrm{CON} ;{ }^{*}$ indicates $\mathrm{p}<0.05$ compared with CON-baseline; $\mathrm{t}$ indicates $\mathrm{p}<0.05$ compared with CON-I/R; $\ddagger$ indicates $\mathrm{p}<0.05$ compared with GDM-baseline group; $\xi$ indicates $\mathrm{p}<0.05$ compared with GDM-I/R; $§$ indicates $\mathrm{p}<0.05$ compared with CON-I/R; a indicates $\mathrm{p}<0.05$ compared with CON-reperfusion. 
Loss of post-I/R cardioprotection is associated with a blunted AMPK response in GDM male offspring rats

To determine whether the increased HW and HW/BW, induced by maternal GDM, affected offspring cardiac function, we first measured the blood pressure (BP) and heart rate (HR) of male offspring. However, there was no difference in the BP or HR between the two groups at 22 weeks of age $(115.40 \pm 1.10 \mathrm{mmHg}$ vs. $110.00 \pm 1.39 \mathrm{mmHg}$, respectively, $\mathrm{n}=8, \mathrm{p}=0.12 ; 376.80 \pm 5.01 \mathrm{bpm}$ vs. $378.20 \pm 11.36 \mathrm{bpm}$, respectively, $\mathrm{n}=8, \mathrm{p}=0.91$ ). We then assessed the contractile function of isolated perfused hearts responding to ischemic insults. The results demonstrated that in the baseline conditions, the hearts from both the control and GDM offspring maintained high levels of cardiac output. However, following a period of $I / R$, recovery was significantly impaired in the GDM group compared with that in the control group ( $63 \%$ vs. $94 \%$, respectively, p<0.05; Fig. 5A).

Immunoblotting of the perfused cardiac tissue demonstrated that the phosphorylation of AMPK and ACC was increased following a period of ischemia and reduced following reperfusion in the hearts from both the control and GDM groups (Fig. 5B,C). In addition, the phosphorylation of AMPK and ACC was significantly lower at both time points in the hearts from the GDM offspring compared to their healthy controls $(\mathrm{p}<0.05)$. There was no significant difference in the Akt pathway; however, Erk (1/2) phosphorylation was significantly compromised in the GDM offspring hearts during ischemia (Fig. 5D,E). Moreover, although a burst of JNK phosphorylation was identified in the GDM group during reperfusion, the difference was not significant (Fig. 5F).

\section{Discussion}

Increasing evidence has demonstrated that GDM significantly impacts not only fetal but also neonatal cardiac structure and function [21, 22]. Despite the establishment of the link between GDM and the development of offspring cardiovascular disease, the underlying mechanism that mediates intrauterine programming remains unclear. In the present study, we used a moderate dose of STZ $(35 \mathrm{mg} / \mathrm{kg})$ to induce hyperglycemia in early-mid pregnancy in rats. Although the STZ model more closely recapitulates hypoinsulinemia rather than the insulin resistance typically observed in GDM, it provided a reliable model to examine the effects of in utero hyperglycemia on fetal programming and development. Moreover, in this GDM model, the maternal weight is lower in the GDM group than in the control during pregnancy, indicating maternal malnutrition, which may profoundly impact offspring cardiac function [23]. Although we did not measure the postpartum weight of the dams, the metabolomic analyses of the postpartum sera suggested that GDM is associated with maternal methionine deficiency. The work of Paul and colleagues on an obese rat model highlighted the potential influence of the maternal gut microbiota composition on maternal and offspring metabolism [24]; moreover, a very recent study in humans suggested that GDM is associated with a disrupted gut microbiota composition, and the differences in the gut microbiota signatures remain detectable at 8 months after pregnancy [25]. These findings indicate that GDM might influence the maternal metabolome through gut microbiota, which, in turn, impact neonatal development through breast-feeding.

Accumulating evidence has demonstrated that female sex hormones protect health in later life, particularly with regard to metabolism and cardiac function [26]. To exclude the confounding influence of sex hormones, we thus focused on the effects of GDM on male offspring. It has been reported that GDM offspring are at a higher risk of being macrosomic [27] and are more likely to be obese in later life [28]. However, Chandna and colleagues reported that GDM offspring had the same weight as controls at birth and in adulthood, whereas they were significantly lighter from 1 week to 3 weeks of age [29]. Yan et al. demonstrated that GDM pups were only heavier than control pups from birth to 3 weeks old [3]. In this study, no differences in weight were identified at birth; however, GDM male offspring were significantly heavier than those from control mothers from 8 weeks of age. A 
similar accelerated growth in GDM offspring has been found in a human study [30]. It could be attributed to maternal malnutrition during pregnancy [31], which may be secondary to hyperglycemia. Despite the differences in body weight, the plasma insulin levels were not different between the GDM and control; moreover, no differences were identified in the measures of offspring glucose and insulin tolerance at 24 weeks of age in this study, which suggests that GDM offspring are not associated with insulin resistance.

We found that the HW and HW/BW ratio were both significantly increased in GDM male offspring at 24 weeks of age, which is consistent with a previously reported correlation between GDM and offspring cardiac hypertrophy [22]. However, the HW and HW/BW were not different in the two groups at birth, which indicates the overgrowth of the heart in the GDM group occurred after delivery. A recent study reported that dietary methionine restriction results in an increased HW/BW ratio in mice [32]. Intriguingly, we reported a reduction of methionine in the GDM dam's metabolome, which is involved in regulating antioxidative enzyme activities in the rat heart [33]. Therefore, GDM associated maternal methionine deficiency could be a causative factor for the abnormal heart growth in male offspring rats; however, the programming effects of in utero hyperglycemia on offspring heart growth also require further evaluation by cross-foster. Moreover, a rodent study has shown that supplementation of a mixture of regulatory amino acids, including methionine, significantly lowered TNF- $\alpha$ production and LDH release during hepatic I/R, suggesting that methionine protects against I/R injury [34]. Methionine labeled with different isotopes has been used for imaging myocardial infarction $[35,36]$, as methionine uptake is substantially elevated in infarcted tissue early after $I / R$, although this finding was initially thought to be associated with macrophage infiltration and inflammation; however, macrophage aggregates at infarcted areas were gradually replaced by newly developed smooth muscle cells [37], which may indicate that methionine is critical for the early acute phase of damage healing, as the initial step in cardiac remodeling. Nevertheless, further assessment of methionine levels in adult male GDM offspring is warranted to ascertain whether it plays a role in GDM programmed susceptibility to cardiac I/R in later life.

Accumulating evidence indicates that compromised AMPK signaling is associated with impaired resistance to ischemia-reperfusion (I/R) injury [38,39]. Therefore, we assumed that AMPK deficiency would lead to a vulnerability to ischemic heart diseases in male offspring of GDM mothers. Male offspring of GDM pregnancies exhibited comparable cardiac contractility in the basal condition; however, they failed to restore cardiac contractile function after acute I/R. When further studies were carried out to determine the effects of GDM on AMPK activity during I/R, it was shown that in addition to decreased AMPK phosphorylation under basal conditions, AMPK activation induced by ischemia was also blunted in male offspring. These results indicate that GDM not only impairs AMPK activity under basal conditions but also inhibits the AMPK response to I/R stress.

AMPK is a key regulator of energy metabolism [40-42], largely through the mediation of fatty acid oxidation via inhibitory phosphorylation of downstream ACC; thus, AMPK plays a crucial role in modulating cardiac fatty acid metabolism $[43,44]$, the predominant energy resource for maintaining normal cardiac function [45]. AMPK phosphorylation is directly regulated by the AMP/ATP ratio through its main upstream regulator LKB1 or CAMKK2 due to increased intracellular $\mathrm{Ca}^{2+}$ [46]. Considering the substantial ATP depletion in cardiac tissue during ischemia, impaired formation of the LKB1/STRAD/MO25 complex is more likely to be the major cause of blunted cardiac AMPK activation in GDM male offspring [47]; however, further investigation of LKB1 is required to fully elucidate the underlying mechanism. Furthermore, it is reported that AMPK regulates the translocation of glucose transporter 4 (GLUT4) to the plasma membrane [48] to facilitate glucose uptake and promote glycolysis [49]. This could supply energy to maintain normal cell function during ischemia and thus avoid the production of a large number of reactive oxygen species during reperfusion through fatty acid $\beta$-oxidation [50]. It is thought that AMPK confers a protective function during I/R via these mechanisms. Further experiments of AMPK activity manipulations in this model are required to validate the role of AMPK activation in mediating GDM-induced intolerance to cardiac insults in male offspring. 


\begin{tabular}{|c|c|}
\hline Cellular Physiology & Cell Physiol Biochem 2019;52:1103-1116 \\
\hline and Biochemistry & \begin{tabular}{l|l} 
DOl: 10.33594/0000000075 & O2019 The Author(s). Published by \\
Published online: 13 April 2019 & Cell Physiol Biochem Press GmbH\&Co. KG
\end{tabular} \\
\hline
\end{tabular}

\section{Conclusion}

This study demonstrated that GDM and associated maternal malnutrition not only programmed a disturbed developmental trajectory in male offspring and thus increased the risk of later life overweight but also resulted in compromised resistance to I/R stress, which was associated with the downregulation of the AMPK signaling pathway. Therefore, an increased risk of cardiovascular disease, such as a greater susceptibility to I/R, remains a risk for individuals born following a pregnancy complicated by GDM.

\section{Acknowledgements}

This work was supported by grants from the National Natural Science Foundation of China (No. 81520108013, 81771613, 81671488, and 81471472), Chongqing Education Committee (CXTDX201601014 and KJ1500223), Chongqing Medical University (CYYQ201507), Commission of Science and Technology of Chongqing Municipality (cstc2017jcyjBX0045), and Chongqing Entrepreneurship and Innovation Supporting Program for Returned Overseas Students (cx2017104). We would also like to acknowledge support from the "111 program" of the Ministry of Education P.R.C. and the State Administration of Foreign Experts Affairs P.R.C.

\section{Disclosure Statement}

The authors declare to have no competing interests.

\section{References}

1 American Diabetes A: Diagnosis and classification of diabetes mellitus. Diabetes Care 2013;36 Suppl 1:S6774.

2 Ferrara A: Increasing prevalence of gestational diabetes mellitus: a public health perspective. Diabetes Care 2007;30 Suppl 2:S141-146.

3 Yan J, Li X, Su R, Zhang K, Yang H: Long-term effects of maternal diabetes on blood pressure and renal function in rat male offspring. PLoS One 2014;9:e88269.

4 Ategbo JM, Grissa O, Yessoufou A, Hichami A, Dramane KL, Moutairou K, Miled A, Grissa A, Jerbi M, Tabka Z, Khan NA: Modulation of adipokines and cytokines in gestational diabetes and macrosomia. J Clin Endocrinol Metab 2006;91:4137-4143.

5 Chou C, Lin C, Yang C, Yang W, Lee F, Tsai M: Pregnancy Outcomes of Taiwanese Women with Gestational Diabetes Mellitus: A Comparison of Carpenter-Coustan and National Diabetes Data Group Criteria. J Womens Health (Larchmt) 2010 19:935-939.

6 Balsells M, García-Patterson A, Gich I, Corcoy R: Major congenital malformations in women with gestational diabetes mellitus: a systematic review and meta-analysis. Diabetes/Metabolism Research and Reviews 2012;28:252-257.

7 Mitanchez D: Foetal and neonatal complications in gestational diabetes: perinatal mortality, congenital malformations, macrosomia, shoulder dystocia, birth injuries, neonatal complications. Diabetes Metab 2010;36:617-627.

8 Poomalar GK: Changing trends in management of gestational diabetes mellitus. World J Diabetes 2015;6:284-295.

9 Saxena P, Tyagi S, Prakash A, Nigam A, Trivedi SS: Pregnancy outcome of women with gestational diabetes in a tertiary level hospital of north India. Indian J Community Med 2011;36:120-123.

10 Thamotharan M, McKnight RA, Thamotharan S, Kao DJ, Devaskar SU: Aberrant insulin-induced GLUT4 translocation predicts glucose intolerance in the offspring of a diabetic mother. Am J Physiol Endocrinol Metab 2003;284:E901-914. 


\section{Cellular Physiology Cell Physiol Biochem 2019;52:1103-1116

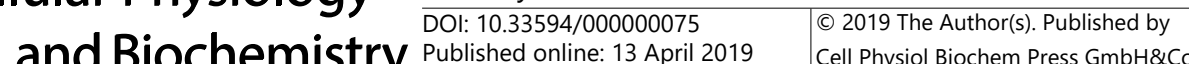 and Biochemistry Published online: 13 April 2019 Cell Physiol Biochem Press GmbH\&Co. KG \\ Luo et al.: GDM Impairs Cardiac AMPK in Male Offspring}

- 11 Cho N, Silverman B, Rizzo T, Metzger B: Correlations between the intrauterine metabolic environment and blood pressure in adolescent offspring of diabetic mothers. J Pediatr 2000;136:587-592.

- 12 Marco LJ, McCloskey K, Vuillermin PJ, Burgner D, Said J, Ponsonby AL: Cardiovascular disease risk in the offspring of diabetic women: the impact of the intrauterine environment. Exp Diabetes Res 2012;2012:565160.

- 13 Arad M, Seidman CE, Seidman JG: AMP-Activated Protein Kinase in the Heart: Role During Health and Disease. Circulation Research 2007;100:474-488.

- 14 Harada M, Nattel SN, Nattel S: AMP-activated protein kinase: potential role in cardiac electrophysiology and arrhythmias. Circ Arrhythm Electrophysiol 2012;5:860-867.

- 15 O’Tierney-Ginn P, Roberts V, Gillingham M, Walker J, Glazebrook PA, Thornburg KL, Grove K, Frias AE: Influence of high fat diet and resveratrol supplementation on placental fatty acid uptake in the Japanese macaque. Placenta 2015;36:903-910.

16 Guo S, Yao Q, Ke Z, Chen H, Wu J, Liu C: Resveratrol attenuates high glucose-induced oxidative stress and cardiomyocyte apoptosis through AMPK. Mol Cell Endocrinol 2015;412:85-94.

17 Song Y, Li J, Zhao Y, Zhang Q, Liu Z, Li J, Chen X, Yang Z, Yu C, Xiao X: Severe maternal hyperglycemia exacerbates the development of insulin resistance and fatty liver in the offspring on high fat diet. Exp Diabetes Res 2012;2012:254976.

- 18 de Seymour JV, Conlon CA, Sulek K, Villas Boas SG, McCowan LM, Kenny LC, Baker PN: Early pregnancy metabolite profiling discovers a potential biomarker for the subsequent development of gestational diabetes mellitus. Acta Diabetol 2014;51:887-890.

- 19 Eleftheriades M, Vafaei H, Dontas I, Vaggos G, Marinou K, Pervanidou P, Sebire NJ, Chrousos GP, Nicolaides $\mathrm{KH}$ : Assessment of body composition in Wistar rat offspring by DXA in relation to prenatal and postnatal nutritional manipulation. Pediatr Res 2016;80:319-325.

20 Tong C, Morrison A, Mattison S, Qian S, Bryniarski M, Rankin B, Wang J, Thomas DP, Li J: Impaired SIRT1 nucleocytoplasmic shuttling in the senescent heart during ischemic stress. FASEB J 2013;27:4332-4342.

21 Arslan D, Oran B, Vatansev H, Cimen D, Guvenc O: The usefulness of plasma asymmetric dimethylarginine (ADMA) levels and tissue doppler echocardiography for heart function in term infants born to mothers with gestational diabetes mellitus. J Matern Fetal Neonatal Med 2013;26:1742-1748.

22 Wang H, Xu Y, Fu J, Huang L: Evaluation of the regional ventricular systolic function by two-dimensional strain echocardiography in gestational diabetes mellitus (GDM) fetuses with good glycemic control. J Matern Fetal Neonatal Med 2015;28:2150-2154.

23 de Belchior AC, Freire DD, Jr., da Costa CP, Vassallo DV, Padilha AS, Dos Santos L: Maternal protein restriction compromises myocardial contractility in the young adult rat by changing proteins involved in calcium handling. J Appl Physiol (1985) 2016;120:344-350.

- 24 Paul HA, Bomhof MR, Vogel HJ, Reimer RA: Diet-induced changes in maternal gut microbiota and metabolomic profiles influence programming of offspring obesity risk in rats. Sci Rep 2016;6:20683.

25 Crusell MKW, Hansen TH, Nielsen T, Allin KH, Ruhlemann MC, Damm P, Vestergaard H, Rorbye C, Jorgensen NR, Christiansen OB, Heinsen FA, Franke A, Hansen T, Lauenborg J, Pedersen O: Gestational diabetes is associated with change in the gut microbiota composition in third trimester of pregnancy and postpartum. Microbiome 2018;6:89.

26 Shah A, Reyes LM, Morton JS, Fung D, Schneider J, Davidge ST: Effect of resveratrol on metabolic and cardiovascular function in male and female adult offspring exposed to prenatal hypoxia and a high-fat diet. J Physiol 2015; DOI:10.1113/JP271133.

27 Yessoufou A, Soulaimann N, Merzouk SA, Moutairou K, Ahissou H, Prost J, Simonin AM, Merzouk H, Hichami A, Khan NA: N-3 fatty acids modulate antioxidant status in diabetic rats and their macrosomic offspring. Int J Obes (Lond) 2006;30:739-750.

28 Boney CM, Verma A, Tucker R, Vohr BR: Metabolic syndrome in childhood: association with birth weight, maternal obesity, and gestational diabetes mellitus. Pediatrics 2005;115:e290-296.

- 29 Chandna AR, Kuhlmann N, Bryce CA, Greba Q, Campanucci VA, Howland JG: Chronic maternal hyperglycemia induced during mid-pregnancy in rats increases RAGE expression, augments hippocampal excitability, and alters behavior of the offspring. Neuroscience 2015;303:241-260.

30 Sovio U, Murphy HR, Smith GC: Accelerated Fetal Growth Prior to Diagnosis of Gestational Diabetes Mellitus: A Prospective Cohort Study of Nulliparous Women. Diabetes Care 2016;39:982-987. 


\section{Cellular Physiology Cell Physiol Biochem 2019;52:1103-1116 \begin{tabular}{ll|l|l} 
DOI: 10.33594/000000075 & C 2019 The Author(s). Published by \\
\hline
\end{tabular} and Biochemistry Published online: 13 April 2019 Cell Physiol Biochem Press GmbH\&Co. KG \\ Luo et al.: GDM Impairs Cardiac AMPK in Male Offspring}

31 Tarry-Adkins JL, Martin-Gronert MS, Fernandez-Twinn DS, Hargreaves I, Alfaradhi MZ, Land JM, Aiken CE, Ozanne SE: Poor maternal nutrition followed by accelerated postnatal growth leads to alterations in DNA damage and repair, oxidative and nitrosative stress, and oxidative defense capacity in rat heart. FASEB J 2013;27:379-390.

32 Ables GP, Ouattara A, Hampton TG, Cooke D, Perodin F, Augie I, Orentreich DS: Dietary methionine restriction in mice elicits an adaptive cardiovascular response to hyperhomocysteinemia. Sci Rep 2015;5:8886.

33 Seneviratne C, Li T, Khaper N, Singal P: Effects of methionine on endogenous antioxidants in the heart. Am J Physiol 1999;277:H2124-2128.

34 Schuster H, Blanc MC, Neveux N, Bonnefont-Rousselot D, Le Tourneau A, De Bandt JP, Cynober L: Protective effects of regulatory amino acids on ischemia-reperfusion injury in the isolated perfused rat liver. Scand J Gastroenterol 2006;41:1342-1349.

35 Morooka M, Kubota K, Kadowaki H, Ito K, Okazaki O, Kashida M, Mitsumoto T, Iwata R, Ohtomo K, Hiroe M: 11C-methionine PET of acute myocardial infarction. J Nucl Med 2009;50:1283-1287.

36 Thackeray JT, Bankstahl JP, Wang Y, Wollert KC, Bengel FM: Targeting Amino Acid Metabolism for Molecular Imaging of Inflammation Early After Myocardial Infarction. Theranostics 2016;6:1768-1779.

37 Taki J, Wakabayashi H, Inaki A, Imanaka-Yoshida K, Hiroe M, Ogawa K, Morooka M, Kubota K, Shiba K, Yoshida T, Kinuya S: 14C-Methionine uptake as a potential marker of inflammatory processes after myocardial ischemia and reperfusion. J Nucl Med 2013;54:431-436.

38 Ma H, Wang J, Thomas DP, Tong C, Leng L, Wang W, Merk M, Zierow S, Bernhagen J, Ren J, Bucala R, Li J: Impaired macrophage migration inhibitory factor-AMP-activated protein kinase activation and ischemic recovery in the senescent heart. Circulation 2010;122:282-292.

39 Wang J, Tong C, Yan X, Yeung E, Gandavadi S, Hare AA, Du X, Chen Y, Xiong H, Ma C, Leng L, Young LH, Jorgensen WL, Li J, Bucala R: Limiting cardiac ischemic injury by pharmacological augmentation of macrophage migration inhibitory factor-AMP-activated protein kinase signal transduction. Circulation 2013;128:225-236.

40 Li J, Qi D, Cheng H, Hu X, Miller EJ, Wu X, Russell KS, Mikush N, Zhang J, Xiao L, Sherwin RS, Young LH: Urocortin 2 autocrine/paracrine and pharmacologic effects to activate AMP-activated protein kinase in the heart. Proc Natl Acad Sci U S A 2013;110:16133-16138.

41 Schisler JC, Rubel CE, Zhang C, Lockyer P, Cyr DM, Patterson C: CHIP protects against cardiac pressure overload through regulation of AMPK. J Clin Invest 2013;123:3588-3599.

- 42 Viollet B, Horman S, Leclerc J, Lantier L, Foretz M, Billaud M, Giri S, Andreelli F: AMPK inhibition in health and disease. Crit Rev Biochem Mol Biol 2010;45:276-295.

- 43 Tong L: Acetyl-coenzyme A carboxylase: crucial metabolic enzyme and attractive target for drug discovery. Cell Mol Life Sci 2005;62:1784-1803.

44 Zhabyeyev P, Gandhi M, Mori J, Basu R, Kassiri Z, Clanachan A, Lopaschuk GD, Oudit GY: Pressure-overloadinduced heart failure induces a selective reduction in glucose oxidation at physiological afterload. Cardiovasc Res 2012;97:676-685.

- 45 Steinberg GR, Kemp BE: AMPK in Health and Disease. Physiol Rev 2009;89:1025-1078.

- 46 Hawley SA, Selbert MA, Goldstein EG, Edelman AM, Carling D, Hardie DG: 5'-AMP activates the AMPactivated protein kinase cascade, and $\mathrm{Ca} 2+$ /calmodulin activates the calmodulin-dependent protein kinase I cascade, via three independent mechanisms. J Biol Chem 1995;270:27186-27191.

47 Hawley SA, Boudeau J, Reid JL, Mustard KJ, Udd L, Makela TP, Alessi DR, Hardie DG: Complexes between the LKB1 tumor suppressor, STRAD alpha/beta and MO25 alpha/beta are upstream kinases in the AMPactivated protein kinase cascade. J Biol 2003;2:28.

48 Lee CT, Ussher JR, Mohammad A, Lam A, Lopaschuk GD: 5'-AMP-activated protein kinase increases glucose uptake independent of GLUT4 translocation in cardiac myocytes. Can J Physiol Pharmacol 2014;92:307314.

49 Wu Y, Sarkissyan M, Mcghee E, Lee S, Vadgama JV: Combined inhibition of glycolysis and AMPK induces synergistic breast cancer cell killing. Breast Cancer Res Treat 2015;151:529-539.

- 50 Balteau M, Van Steenbergen A, Timmermans AD, Dessy C, Behets-Wydemans G, Tajeddine N, CastanaresZapatero D, Gilon P, Vanoverschelde JL, Horman S, Hue L, Bertrand L, Beauloye C: AMPK activation by glucagon-like peptide-1 prevents NADPH oxidase activation induced by hyperglycemia in adult cardiomyocytes. Am J Physiol Heart Circ Physiol 2014;307:H1120-1133. 